MADPH-01-1241

hep-ph/0108261

\title{
Testing radiative neutrino mass generation via $R$-parity violation at the Tevatron
}

\author{
V. Barger, T. Han, S. Hesselbach and D. Marfatia \\ Department of Physics, University of Wisconsin, Madison, WI 53706, USA
}

\begin{abstract}
An $R$-parity violating SUSY model with lepton-number violating couplings $\lambda_{i 33}^{\prime}, i=2,3$ can generate a neutrino mass spectrum that explains the recent results from neutrino oscillation experiments. These $R$-parity violating couplings lead to a clean signal with at least one isolated lepton and at least three tagged $b$ jets that is accessible in chargino and neutralino production at the Tevatron collider. This signature can be probed at $3 \sigma$ up to $m_{1 / 2}=230 \mathrm{GeV}(320 \mathrm{GeV})$ with an integrated luminosity of $2 \mathrm{fb}^{-1}\left(30 \mathrm{fb}^{-1}\right)$.

PACS numbers: 12.60.Jv., 14.60.Ly, 14.80.Pq
\end{abstract}

There is now strong evidence from both atmospheric [1] and solar [2,3] neutrino experiments that neutrinos are not massless. The $\nu_{\mu}$ flux deficit and zenith angle dependence seen in atmospheric experiments is well-explained if $\nu_{\mu} \rightarrow \nu_{\tau}$ oscillations occur with maximal mixing and $\delta m^{2} \sim 3.5 \times 10^{-3} \mathrm{eV}^{2}$. The recent results from the SNO experiment [3], give compelling confirmation that $\nu_{e}$ neutrinos from the Sun oscillate to $\nu_{\mu, \tau}$ neutrinos with large mixing and quite possibly $\delta m^{2} \sim 5 \times 10^{-5} \mathrm{eV}^{2}$ 伯. Thus, it is important to find a testable explanation of these mixing angles and mass-squared differences. The most promising extension of the Standard Model is obtained by its supersymmetrization. In the Minimal Supersymmetric Standard Model (MSSM) a discrete symmetry called $R$-parity is usually assumed to be conserved. However, if this discrete symmetry is broken, radiative neutrino mass generation is a direct consequence of the MSSM [5]. In this Letter, we show that this alternative to the see-saw mechanism [6] can be subject to direct tests at the Tevatron collider.

Radiative neutrino mass generation

The most general superpotential allowed by SM gauge symmetry and supersymmetry contains the explicit $R$ parity violating terms

$$
W_{\not R}=\lambda_{i j k} L_{i} L_{j} \bar{E}_{k}+\lambda_{i j k}^{\prime} L_{i} Q_{j} \bar{D}_{k}+\lambda_{i j k}^{\prime \prime} \bar{U}_{i} \bar{D}_{j} \bar{D}_{k},
$$

where $i, j, k=1,2,3$ are generation indices and $L, Q$, $E, D$ and $U$ denote the left-handed lepton and quark doublets and the right-handed charged-lepton down- and up-type quark singlets, respectively [7]. The first two terms $\left(\lambda, \lambda^{\prime}\right)$ violate lepton-number and the third term $\left(\lambda^{\prime \prime}\right)$ baryon-number conservation.

An important consequence of the $\lambda^{\prime}$ interactions is the generation of Majorana masses for left-handed neutrinos through quark-squark loops as in Fig. 1. Similar diagrams result from the $\lambda$ couplings. The dominant contribution to neutrino masses comes from $b \tilde{b}$ loops, so we focus on the $R$-parity breaking interactions resulting from $\lambda_{i 33}^{\prime}$ :

$$
\mathcal{L} / \lambda_{i 33}^{\prime}=\tilde{\nu}_{L}^{i} \bar{b}_{R} b_{L}+\tilde{b}_{L} \bar{b}_{R} \nu_{L}^{i}+\left(\tilde{b}_{R}\right)^{*}\left(\bar{\nu}_{L}^{i}\right)^{c} b_{L}-\tilde{e}_{L}^{i} \bar{b}_{R} t_{L}
$$

$$
-\tilde{t}_{L} \bar{b}_{R} e_{L}^{i}-\left(\tilde{b}_{R}\right)^{*}\left(\bar{e}_{L}^{i}\right)^{c} t_{L}+\text { h.c. }
$$

Specific models involving $\lambda_{i 33}^{\prime}$ that accommodate maximal $\nu_{\mu}-\nu_{\tau}$ mixing in atmospheric neutrino oscillations and the Large Mixing Angle solution to the solar neutrino anomaly have been constructed in Ref. [8]. The mass matrix arising from these models takes the form

$$
m_{\nu} \sim \frac{3}{8 \pi^{2}} \lambda^{\prime 2} \frac{m_{b}^{2}}{m_{\tilde{q}}}\left(\begin{array}{ccc}
\epsilon^{2} & \epsilon & \epsilon \\
\epsilon & 1 & 1 \\
\epsilon & 1 & 1
\end{array}\right)
$$

where $\epsilon=m_{s} / m_{b}$. The explicit breaking of a discrete symmetry that automatically forbids baryon number violating interactions and bilinear $R$-parity violating couplings leads to a suppression of the $\lambda_{133}^{\prime}$ coupling by $\epsilon$ relative to $\lambda_{233}^{\prime}$ and $\lambda_{333}^{\prime}$. Although the bilinear terms are generated at one-loop, their contributions to the neutrino mass matrix of Eq. (3) are estimated to be small, suppressed by a loop-factor squared. With

$$
\lambda_{233}^{\prime} \simeq \lambda_{333}^{\prime} \sim 10^{-4}\left(\frac{m_{\tilde{q}}}{200 \mathrm{GeV}}\right)^{1 / 2},
$$

the largest mass is $m_{\nu_{3}} \sim 0.06 \mathrm{eV}$, corresponding to the atmospheric scale $\sqrt{\delta m_{\text {atm }}^{2}}$ [1]. The values of these couplings are well within the existing $2 \sigma$ limits: $\lambda_{233}^{\prime}, \lambda_{333}^{\prime}$ of $\mathcal{O}(0.1)$ and $\lambda_{133}^{\prime}$ of $\mathcal{O}\left(10^{-3}\right)$ [9]. The two other eigenvalues of the matrix in Eq. (3) are shifted from zero by corrections of $\mathcal{O}(\epsilon)$, thereby yielding the correct ratio for $\delta m_{\text {solar }}^{2} / \delta m_{\text {atm }}^{2} \sim \epsilon^{2}$.

Production and decay of the LSP

Because the $R$-parity violating Yukawa couplings are small, we assume the sparticles have the same mass patterns as in the mSUGRA model [10] and the lightest neutralino $\left(\tilde{\chi}_{1}^{0}\right)$ is the lightest supersymmetric particle (LSP). The production and decay of the sparticles occur dominantly through the $R$-parity conserving channels except for the decays of the LSP.

The dominant SUSY production processes for heavy squarks and gluinos at the Fermilab Tevatron are pair 


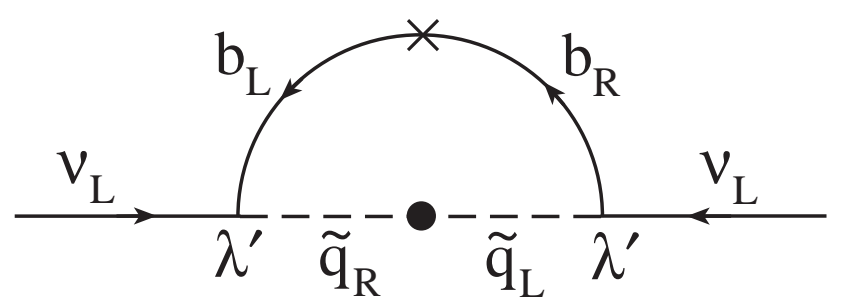

FIG. 1. The dominant one loop diagram via which Majorana mass terms are generated for left-handed neutrinos.

production of the lightest chargino $\left(q \bar{q} \rightarrow \tilde{\chi}_{1}^{+} \tilde{\chi}_{1}^{-}\right)$and associated production of the lightest chargino with the two lightest neutralinos $\left(q \bar{q}^{\prime} \rightarrow \tilde{\chi}_{1}^{ \pm} \tilde{\chi}_{i}^{0}, i=1,2\right)[10]$. The $\tilde{\chi}_{1}^{ \pm}$and $\tilde{\chi}_{2}^{0}$ decay in their $R$-parity conserving channels into the LSP.

Figure 2 shows contours of the rest decay length $c \tau$ (in meters) of the LSP in the $m_{0}-m_{1 / 2}$ parameter space for $\lambda_{233}^{\prime}=\lambda_{333}^{\prime}=10^{-4}$. Since $\lambda_{133}^{\prime}$ is two orders of magnitude smaller than $\lambda_{233}^{\prime}$ and $\lambda_{333}^{\prime}$, its effect on the branching ratio of the LSP can be safely ignored. Except in scenarios with $m_{0} \gg m_{1 / 2}$, the LSP decays well inside the detector. In general the decay length scales according to $c \tau \sim\left(\lambda_{i 33}^{\prime}\right)^{-2}$. For $m_{\tilde{\chi}_{1}^{0}}<m_{t}+m_{b}$, the LSP decays to $\tilde{\nu}_{i}^{*} \nu_{i}$ and $\tilde{b}^{*} b$ lead to a neutrino and a $b \bar{b}$ pair:

$$
\tilde{\chi}_{1}^{0} \rightarrow \nu_{\mu} b \bar{b}, \nu_{\tau} b \bar{b}+\text { c.c. }
$$

For $m_{\tilde{\chi}_{1}^{0}}>m_{t}+m_{b}$, the decay channels involving a lepton and a top quark are also open $\left(\tilde{\chi}_{1}^{0} \rightarrow \nu_{\mu} b \bar{b}, \nu_{\tau} b \bar{b}, \mu t \bar{b}, \tau t \bar{b}+\right.$ c.c.). For $m_{1 / 2} \lesssim 500 \mathrm{GeV}$ the top channels have no appreciable branching fraction and even for $m_{1 / 2}<$ $1000 \mathrm{GeV}$ their branching fractions are always smaller than $\sim 20 \%$. Hence we focus on the decay of the LSP into a neutrino and a $b \bar{b}$ pair. Since the sparticles are produced in pairs and decay into the LSP through the usual $R$-parity conserving channels, there are two $\tilde{\chi}_{1}^{0}$ LSPs in each supersymmetric event. Thus we have the promising signature of four $b$ jets and missing energy.

Signals and SM backgrounds

We simulate SUSY events at the parton level with the event generator PYTHIA, version 6.157, which includes the supersymmetric extension SPYTHIA [11] and a further extension which provides the $R$-parity violating decays of the LSP [12]. The masses of the SUSY particles are computed from the mSUGRA input parameters $m_{0}$, $m_{1 / 2}, \tan \beta, A_{0}$ and $\operatorname{sign}(\mu)$ using the two loop RGEs of the program ISAJET 13 and then used as electroweak scale input parameters for PYTHIA.

The detector energy resolutions at the Tevatron are simulated with 14

$$
\begin{array}{ll}
\Delta E_{j} / E_{j}=0.8 / \sqrt{E}_{j} & \text { for hadrons, } \\
\Delta E_{e} / E_{e}=0.2 / \sqrt{E}_{e} & \text { for electrons },
\end{array}
$$

and the smearing of muons is neglected. We add the smeared transverse momenta of all the particles to obtain

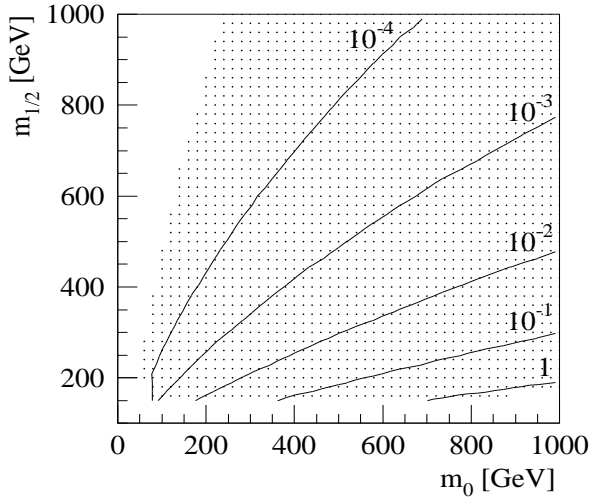

FIG. 2. Contours of the rest decay length $c \tau$ (in meters) of the LSP in the $m_{0}-m_{1 / 2}$ parameter space for $\lambda_{233}^{\prime}=\lambda_{333}^{\prime}=10^{-4}$. The other parameters are fixed at $A_{0}=0, \tan \beta=10$ and $\mu>0$. The dots represent mSUGRA points that satisfy $m_{\tilde{\chi}_{1}^{ \pm}}, m_{\tilde{e}_{R}}>100 \mathrm{GeV}, m_{\tilde{\tau}_{1}}>85 \mathrm{GeV}$ and $m_{h}>90 \mathrm{GeV}$.

the missing transverse momentum $\not_{T}$ of the event. Then we apply the "basic cuts"

$$
p_{T}>15 \mathrm{GeV}, \quad|\eta|<2, \quad \Delta R>0.5
$$

on the $b$ and $c$ quarks in the event and simulate the $b$ tagging efficiency by accepting the surviving $b$ quarks with $50 \%$ probability and the misidentification of $c$ by accepting the surviving $c$ quarks with $5 \%$ probability as $b$ jets. We ensure the isolation of the $b$ jets by counting two $b$ with $\Delta R_{b b}<0.5$ as one $b$ jet. To take advantage of the large number of $b$ quarks in the SUSY events and to suppress the background, we consider two signatures with at least three tagged $b$ jets and

1. at least one isolated lepton $(l=e, \mu)$ which survives the basic cuts $p_{T}>10 \mathrm{GeV},|\eta|<2$ $(\geq 3 b, \geq 1 l$ signature) or

2. a missing transverse momentum $\not_{T}>20 \mathrm{GeV}$ $\left(\geq 3 b, p_{T}\right.$ signature).

The lepton isolation is ensured by counting only leptons with no other particle in a cone $\Delta R<0.5$ around the lepton. The $\geq 3 b, p_{T}$ signature has the advantage that all the supersymmetric production processes contribute regardless of the particles produced and their decays.

The major background process is $t \bar{t}$ production where one of the $W$ decays via $W^{-} \rightarrow b \bar{q}, \quad(q=c, u)$ or a $c$ quark from $W^{-} \rightarrow s \bar{c}$ is misidentified. The branching ratio of the former decay is very small so the background from the latter decay and misidentified $c$ dominates. There is, in addition, a potentially dangerous background for the $\geq 3 b, p_{T}$ signal from the QCD production of $b \bar{b} b \bar{b}, b \bar{b} c \bar{c}$. With the basic cuts of Eq.(7), the background rate from this process with three tagged $b$ jets is about $5 \mathrm{pb}$, which is more than an order of magnitude higher than our typical signal rate. Although there 

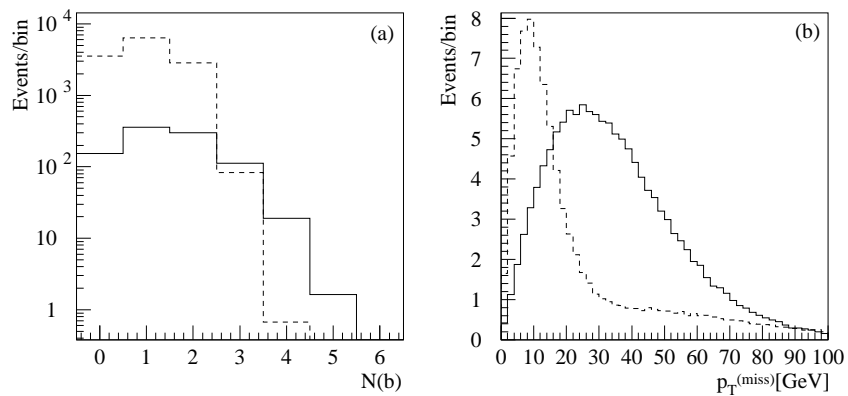

FIG. 3. Distributions (a) of the number of tagged $b$ jets and (b) of the missing transverse energy $\not_{T}$ in events with at least three tagged $b$ jets for the SUSY production processes in the reference scenario of Eq. (8) (solid) and for the $t \bar{t}$ background (dashed). The distributions are normalized to the expected number of events at Run II of the Tevatron with $2 \mathrm{fb}^{-1}$.

is no significant $\not_{T}$ from the process, the imperfect jet energy measurement and $b$ semileptonic decay can result in some $\not_{T}$. However, we expect that our cut $p_{T}>20 \mathrm{GeV}$ and other kinematical cuts would significantly suppress this reducible QCD background. More detailed simulations of this background to the $\geq 3 b, p_{T}$ signal need to be implemented.

There are kinematical differences between the signal and the $t \bar{t}$ background that can be exploited to separate them. In particular, the distributions of the number of $b$ jets and transverse momenta are different for the signal and background. For purposes of illustration, we choose an mSUGRA reference scenario

$m_{1 / 2}=m_{0}=200 \mathrm{GeV}, A_{0}=0, \tan \beta=10, \mu>0$,

for which the masses of the neutralinos and charginos are

$m_{\tilde{\chi}_{1}^{0}}=75 \mathrm{GeV}, m_{\tilde{\chi}_{2}^{0}}=135 \mathrm{GeV}, m_{\tilde{\chi}_{1}^{+}}=133 \mathrm{GeV}$.

Figure $3(\mathrm{a})$ shows the distribution of the number of tagged $b$ jets from the $R$-parity violating decay of the LSP in the reference scenario of Eq. (8) and from the $t \bar{t}$ background. Here and henceforth, we represent the signal with a solid line and the background with a dashed line. While the background for events with 0,1 or 2 tagged $b$ jets is more than 10 times as large as the SUSY signal, the requirement of at least three tagged $b$ jets yields a signal-to-background event ratio $S / B=133 / 84$ for the expected luminosity $2 \mathrm{fb}^{-1}$ of the Tevatron Run II even without further cuts.

Figure 3(b) gives the distributions of the missing energy $p_{T}$ in events with at least three tagged $b$ jets. The background is peaked for low missing energy, hence the cut $p_{T}>20 \mathrm{GeV}$ significantly suppresses the background in the $\geq 3 b, \not_{T}$ signature, yielding $S / B=100 / 31$.

In Fig. 目, we show the distribution of the missing transverse energy and the transverse momentum of the hardest $b$ jet in events with at least three tagged $b$ jets and at least one isolated lepton. The $p_{T}$-distribution of the hardest $b$
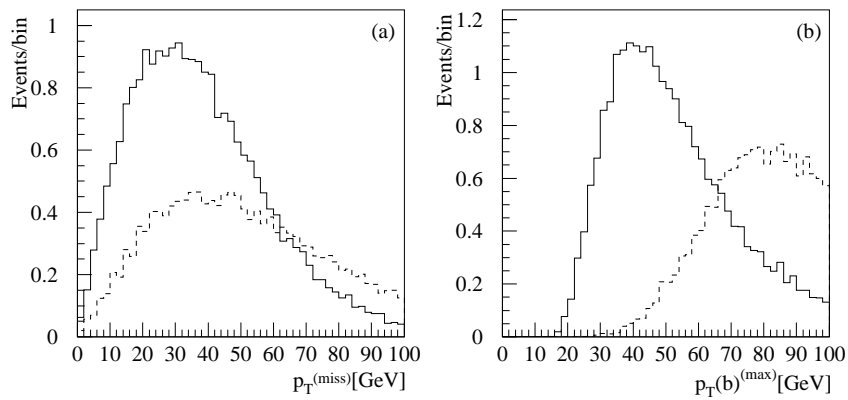

FIG. 4. Distributions (a) of the missing transverse energy $p_{T}$ and (b) of the transverse momenta of the hardest $b$ jet in events with at least $3 b$ jets and at least 1 isolated lepton $(\geq 3 b, \geq 1 l$ signature).

shows that a more restrictive $p_{T}$ cut would bring no improvement. An upper cut on $p_{T}(b)$ enhances the signalto-background ratio from $S / B=23 / 15$ without cut to $S / B=19 / 3$ with $p_{T}(b)<70 \mathrm{GeV}$ for $2 \mathrm{fb}^{-1}$. This cut also slightly improves the minimum required luminosity for detection of the signal.

Figure 5 shows the transverse momenta of the third hardest tagged $b$ jet and of the hardest jet in events of the $\geq 3 b, p_{T}$ signature. The distribution of the third hardest $b$ in the SUSY signal is peaked at low $p_{T}$, so a lower cut on the transverse momentum of the $b$ jets other than the basic cut $p_{T}>15 \mathrm{GeV}$ is not useful for this signature, too. But the transverse momentum of the hardest $b$ of the background is significantly higher than in the SUSY signal. Hence an upper cut on $p_{T}$ can improve the signal-to-background ratio; for $2 \mathrm{fb}^{-1}, S / B=100 / 31$ without a cut and $S / B=76 / 5$ with an additional cut, $p_{T}(b)<70 \mathrm{GeV}$.

Discussion and conclusion

The regions in the $m_{0}-m_{1 / 2}$ parameter space where the two signatures yield $3 \sigma$ and $5 \sigma$ evidence are shown in Fig. 6. The signals depend mainly on $m_{1 / 2}$ and only weakly on $m_{0}$ because $m_{1 / 2}$ determines the masses of the neutralinos and charginos produced (see e.g. Ref. [15]).

For large $m_{0} \gtrsim 100 \mathrm{GeV}$ the $3 \sigma(5 \sigma)$ reach of Tevatron Run II with $2 \mathrm{fb}^{-1}$ is $m_{1 / 2} \approx 230 \mathrm{GeV}(190 \mathrm{GeV})$ for the
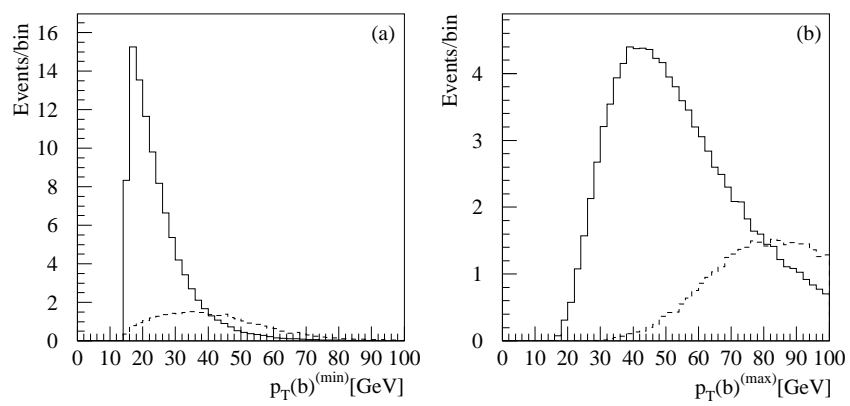

FIG. 5. Distributions of the transverse momenta (a) of the third hardest $b$ jet and (b) of the hardest jet in events with at least $3 b$ jets and $\not p_{T}>20 \mathrm{GeV}\left(\geq 3 b, p_{T}\right.$ signature). 


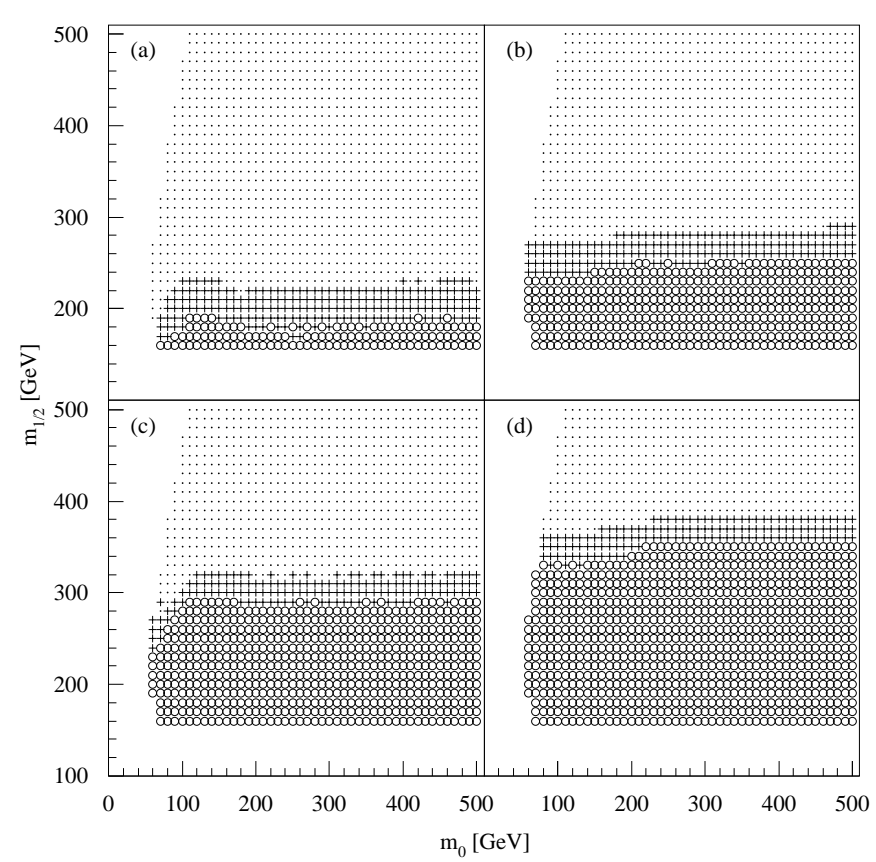

FIG. 6. Regions in the $m_{0}-m_{1 / 2}$ parameter space where the signatures give a $3 \sigma$ (pluses) and $5 \sigma$ (circles) signal: (a) ( $\geq 3 b$, $\geq 1 l)$ with $2 \mathrm{fb}^{-1}$, (b) $\left(\geq 3 b, p_{T}>20 \mathrm{GeV}\right)$ with $2 \mathrm{fb}^{-1}$, (c) $(\geq 3 b, \geq 1 l)$ with $30 \mathrm{fb}^{-1}$ and $(\mathrm{d})\left(\geq 3 b, p_{T}>20 \mathrm{GeV}\right)$ with $30 \mathrm{fb}^{-1}$. The other parameters are fixed at $A_{0}=0, \tan \beta=10$ and $\mu>0$. The dots show mSUGRA points that satisfy $m_{\tilde{\chi}_{1}^{ \pm}}, m_{\tilde{e}_{R}}>100 \mathrm{GeV}, m_{\tilde{\tau}_{1}}>85 \mathrm{GeV}$ and $m_{h}>90 \mathrm{GeV}$.

signature with at least $3 b$ jets and at least one isolated lepton. A luminosity of $30 \mathrm{fb}^{-1}$ can improve this reach to $m_{1 / 2} \approx 320 \mathrm{GeV}(290 \mathrm{GeV})$. In the signature with at least $3 b$ jets and $\not p_{T}>20 \mathrm{GeV}$, the signal is potentially larger. The $3 \sigma(5 \sigma)$ reach is $m_{1 / 2} \approx 290 \mathrm{GeV}(250 \mathrm{GeV})$ for $2 \mathrm{fb}^{-1}$ which improves to $380 \mathrm{GeV}(350 \mathrm{GeV})$ for $30 \mathrm{fb}^{-1}$.

In summary, we have demonstrated that neutrino mass generation via $R$-parity violating SUSY can be tested at the Tevatron collider. The broken $R$-parity interactions are a simple and natural way of generating neutrino masses and provide a theoretical alternative to the see-saw mechanism. The neutrino masses and mixings indicated by the experimental results on neutrino oscillations can be explained with $\lambda_{133}^{\prime} \ll \lambda_{i 33}^{\prime} \sim 10^{-4}$, $i=2,3$. Because of the smallness of these couplings, the production and decay of sparticles proceeds via the $R$-parity conserving channels except for the decay of the LSP which decays into a $b \bar{b}$ pair and a neutrino in most of the SUSY parameter space. At the Tevatron collider, the clean signature of at least three tagged $b$ jets and at least one isolated lepton can be probed at $3 \sigma$ up to $m_{1 / 2} \approx 230 \mathrm{GeV}(320 \mathrm{GeV})$ with $2 \mathrm{fb}^{-1}\left(30 \mathrm{fb}^{-1}\right)$. Another possible signature with at least three tagged $b$ jets and missing energy $p_{T}>20 \mathrm{GeV}$ may improve the reach up to $m_{1 / 2} \approx 290 \mathrm{GeV}(380 \mathrm{GeV})$ with $2 \mathrm{fb}^{-1}\left(30 \mathrm{fb}^{-1}\right)$ at $3 \sigma$. The physics reach in these channels is summarized in Fig. 6 .

Acknowledgments: We thank S. Dasu and S. Pakvasa for discussions. This research was supported by the U.S. DOE under Grant No. DE-FG02-95ER40896 and by the WARF. S.H. is supported by DFG under contract No. HE 3241/1-1.

[1] Y. Fukuda et al., Phys. Lett. B467, 185 (1999); W. Allison et al., Phys. Lett. B449, 137 (1999); M. Ambrosio et al., hep-ex/0106049.

[2] B. Cleveland et al., Astropart. Phys. 496, 505 (1998); J. Abdurashitov et al., Phys. Rev. C60, 055801 (1999); W. Hampel et al., Phys. Lett. B447, 127 (1999); M. Altmann et al., Phys. Lett. B490, 16 (2000); S. Fukuda et al., hep-ex/0103032.

[3] Q. Ahmad et al., nucl-ex/0106015.

[4] V. Barger et al., hep-ph/0106207, G.L. Fogli et al., hep-ph/0106247, J. Bahcall et al., hep-ph/0106258; A. Bandyopadhya et al., hep-ph/0106264.

[5] C. S. Aulakh and R. N. Mohapatra, Phys. Lett. 119B, 136 (1982); L. Hall and M. Suzuki, Nucl. Phys. B231, 419 (1984); J. Ellis et al., Phys. Lett. 150B, 142 (1985); G. Ross and J. Valle, Phys. Lett. 151B, 375 (1985); S. Dawson, Nucl. Phys. B261, 297 (1985).

[6] M. Gell-Mann, P. Ramond and R. Slansky, in Supergravity, eds. P. van Niewenhuizen and D. Freedman (North Holland 1979); T. Yanagida, in Proceedings of Workshop on Unified Theory and Baryon number in the Universe, eds. O. Sawada and A. Sugamoto (KEK 1979); R. N. Mohapatra and G. Senjanovic, Phys. Rev. Lett. 44, 912 (1980).

[7] V. Barger, G.F. Giudice and T. Han, Phys. Rev. D40, 2987 (1989); for reviews, see e.g., H. Dreiner, in Perspectives on supersymmetry, Ed. G.L. Kane (World Scientific) p. 462, hep-ph/9707435 and G. Bhattacharyya, hep-ph/9709395.

[8] M. Drees, S. Pakvasa, X. Tata and T. ter Veldhuis, Phys. Rev. D57, 5335 (1998).

[9] B. Allanach et al., contribution to Physics at Run II: Workshop on Supersymmetry/Higgs, hep-ph/9906224; O. Lebedev, W. Loinaz and T. Takeuchi, Phys. Rev. D62, 015003 (2000).

[10] S. Abel et al., contribution to Physics at Run II: Workshop on Supersymmetry/Higgs, hep-ph/0003154.

[11] T. Sjöstrand, Comput. Phys. Commun. 82, 74 (1994); S. Mrenna, Comput. Phys. Commun. 101, 232 (1997); T. Sjöstrand et al., Comput. Phys. Commun. 135, 238 (2001).

[12] P. Paganini, CMS-IN-2000/019.

[13] H. Baer, F. Paige, S. Protopopescu and X. Tata, hep$\mathrm{ph} / 0001086$.

[14] M. Carena et al., contribution to Physics at Run II: Workshop on Supersymmetry/Higgs, hep-ph/0010338.

[15] V. Barger and C. Kao, Phys. Rev. D57, 3131 (1998). 